\title{
BMJ Open Study protocol: a multicentre, prospective, phase II trial of isotoxic hypofractionated concurrent chemoradiotherapy for non-small cell lung cancer
}

\author{
Yue-E Liu, ${ }^{1}$ Xiao-Ying Xue ${ }^{2}$ Rui Zhang, ${ }^{1}$ Xue-Ji Chen, ${ }^{1}$ Yu-Xia Ding, ${ }^{1}$ \\ Chao-Xing Liu, ${ }^{3}$ Yue-Liang Qin, ${ }^{1}$ Wei-Qian Li, ${ }^{1}$ Xiao-Cang Ren, ${ }^{1}$ Qiang Lin (D) ${ }^{1,4}$
}

To cite: Liu Y-E, Xue X$\mathrm{Y}$, Zhang R, et al. Study protocol: a multicentre, prospective, phase II trial of isotoxic hypofractionated concurrent chemoradiotherapy for non-small cell lung cancer. BMJ Open 2020;10:e036295. doi:10.1136/ bmjopen-2019-036295

- Prepublication history and additional material for this paper are available online. To view these files, please visit the journal online (http://dx.doi org/10.1136/bmjopen-2019036295).

Received 14 December 2019 Revised 08 September 2020 Accepted 09 October 2020

\section{Check for updates}

(c) Author(s) (or their employer(s)) 2020. Re-use permitted under CC BY-NC. No commercial re-use. See rights and permissions. Published by BMJ.

${ }^{1}$ Department of Oncology, North China Petroleum Bureau General Hospital, Hebei Medical University, Renqiu, Hebei, China ${ }^{2}$ Department of Radiotherapy, Second Hospital of Hebei Medical University, Shijiazhuang, Hebei, China

${ }^{3}$ Department of Oncology, No.1 Hospital of Shijiazhuang City,

Shijiazhuang, Hebei, China

${ }^{4}$ Hebei Medical University, Shijiazhuang, Hebei, China

Correspondence to Professor Qiang Lin; billhappy001@163.com

\section{ABSTRACT}

Introduction Concurrent chemoradiotherapy with conventional fractionation has been acknowledged as one of the standard treatments for locally advanced non-small cell lung cancer (NSCLC). The radiotherapy dose of 60 Gy is far from enough for local tumour control. Due to this fact, hypofractionated radiotherapy can shorten the total treatment duration, partially counteract the accelerated repopulation of tumour cells and deliver a higher biological effective dose, it has been increasingly used for NSCLC. In theory, concurrent hypofractionated chemoradiotherapy can result in an enhanced curative effect. To date, the vast majority of radiotherapy prescriptions assign a uniform radiotherapy dose to all patients. However this kind of uniform radiotherapy prescription may lead to two consequences: excess damage to normal tissues for large tumours and insufficient dose for small tumours. Our study aims to evaluate whether delivering individualised radiotherapy dose is feasible using intensity-modulated radiotherapy.

Methods and analysis Our study of individualised radiotherapy is a multicenter phase II trial. From April 2019, a total of 30 patients from three Chinese centres, with a proven histological or cytological diagnosis of inoperable NSCLC, will be recruited. The dose of radiation will be increased until one or more of the organs at risk tolerance or the maximum dose of 69 Gy is reached.

The primary end point is feasibility, with response rates, progression-free survival and overall survival as secondary end points. The concurrent chemotherapy regimen will be docetaxel plus lobaplatin.

Ethics and dissemination The study has been approved by medical ethics committees from three research centres. The trial is conducted in accordance with the Declaration of Helsinki.

The trial results will be disseminated through academic conference presentations and peer-reviewed publications. Trial registration number NCT03606239

\section{INTRODUCTION}

Lung cancer ranks first worldwide among all malignant tumours in terms of incidence and mortality rates, while non-small cell lung

\section{Strengths and limitations of this study}

- This is a prospective multicentre study.

- Radiotherapy is delivered using hypofractionation and individualised doses are prescribed to all patients.

- Unified quality control is applied

- Radiotherapy is delivered using four-dimensional CT simulation only when it is available.

- This is a single-arm study.

cancer (NSCLC) accounts for 80\%-85\% of lung cancers. ${ }^{1}$ Concurrent chemoradiotherapy has been proven to be one of the standard treatments for locally advanced NSCLC. ${ }^{2}$ At present, a conventionally fractionated dose of $60 \mathrm{~Gy}$ is still recommended as the standard radiotherapy dose for concurrent chemoradiotherapy. ${ }^{3}$ A model study has shown that in the conventional fractionation mode, a dose of $84 \mathrm{~Gy}$ is required to achieve local control for more than 30 months. ${ }^{4}$ Moreover, it has been confirmed in the clinical application of stereotactic body radiation therapy (SBRT) that a biological effective dose (BED) of at least $100 \mathrm{~Gy}$ is required for stage I NSCLC. ${ }^{5}$ Therefore, a conventionally fractionated dose of $60 \mathrm{~Gy}$, that is, the so-called 'standard dose', cannot achieve satisfactory local tumor control. The Radiation Therapy Oncology Group (RTOG) conducted a radiotherapy dose escalation test. The phase I/II trial showed that a dose of $74 \mathrm{~Gy}$ was safe and effective. ${ }^{6}$ However, the results of the prospective, multicenter, randomised controlled study RTOG 0617 were disappointing. The high radiation dose (74Gy) failed to improve the survival of the patients and increased the toxicity. ${ }^{3}$ One of the possible reasons that the high dose did 
not enhance survival is that the total treatment duration was overly long. ${ }^{7}$ One study reported that NSCLC cells began to undergo accelerated repopulation at 3 weeks after the start of radiotherapy, and an additional daily dose of $0.6 \mathrm{~Gy}$ was required to compensate for the loss of local control. ${ }^{8}$ Conventional fractionation of $74 \mathrm{~Gy}$ took 7.4 weeks to complete, which was 11 days longer than fractionation of $60 \mathrm{~Gy}$. Therefore, conventional fractionation of $74 \mathrm{~Gy}$ may not be effective for improving BED. Hypofractionated radiotherapy shortens the total treatment duration and partially offsets the accelerated repopulation effect, thereby delivering high-dose radiation to the tumours in a short period of time, improving BED and strengthening local tumour control. In theory, hypofractionated radiotherapy concurrently combined with chemotherapy can enhance the curative effect. ${ }^{9}$ However, hypofractionated concurrent chemoradiotherapy increases adverse reactions. Therefore, balancing efficacy and safety to identify the appropriate radiotherapy dose is an urgent issue to address. Previously, we conducted a phase I/II clinical trial on hypofractionated radiotherapy (3 Gy/fraction) concurrent with chemotherapy. During the phase I trial, we recommended $69 \mathrm{~Gy}$ as the maximum tolerated dose (MTD), which was concurrently combined with vinorelbine and carboplatin in the phase II trial. ${ }^{10}$ However, only 12 patients were enrolled before the early termination of the trial. One of the important reasons was the severe, intolerable radiation-induced oesophagitis. ${ }^{11}$ After summarising and comparing the enrolled patients and the radiotherapy dose parameters in the phase I and phase II trials, we believe that administration of a uniform radiotherapy dose to all patients may give rise to the following two consequences due to tumour size differences and adjacent organs: First, for large tumours and/or tumours adjacent to important tissues, severe radiotherapy damage may occur, which is likely to prevent patients from completing the radiotherapy regimen. Second, for small tumours that are not adjacent to important organs, the radiotherapy dose may be insufficient, which causes a decrease in local control, and thus affects efficacy. Haslett $e t a l^{12}$ had launched a trial using 'isotoxic intensity-modulated radiotherapy (IMRT)', and IMRT was delivered with hyperfractionated radiotherapy without concurrent chemotherapy. The trial had completed recruitment of 35 patients in 2016. Radiotherapy doses all patients received were individually escalated until one or more of the organs at risk (OAR) tolerance or the maximum dose of $79.2 \mathrm{~Gy}$ was reached. This trial preliminarily proved the isotoxic radiotherapy was feasible. Our trial employs similar methods of isotoxic, individualised radiotherapy prescriptions to give patients the highest possible radiotherapy dose within normal tissue tolerance. Such an individualised prescription dose guided by the concept of 'isotoxicity' actually achieves radiotherapy dose escalation for tumours. ${ }^{13}$ The MTDs of normal tissue for lung cancer radiotherapy is mainly based on conventional fractionated irradiation. No guidelines of MTDs for normal tissues used in moderate hypofractionated radiotherapy are available. ${ }^{14}$ Since it is the first time that we have applied isotoxic dose escalation to hypofractionated radiotherapy, we have no standard that can be applied directly. We mainly refer to the MTDs of normal tissues in the published literature of hypofractionated radiotherapy, ${ }^{14-18}$ in addition to a combination of former clinical trials of hypofractionated concurrent chemoradiotherapy that we have previously carried out. ${ }^{1011}$ The MTD was set for normal tissues in this study based on above data. In order to further ensure the safety of patients, as far as possible, while avoiding serious injury to patients caused by the inappropriate MTD of normal tissue, we also have considerations in the statistical design (details in Study Design). The set values will be verified in this study. To our knowledge, this is the first study in the world that applies the concept of isotoxicity in concurrent hypofractionated radiotherapy and chemotherapy to provide patients with an individualised radiotherapy dose.

\section{METHODS}

\section{Research goals}

The primary endpoint of the present study is to explore the safety of an individualised radiotherapy dose in isotoxic hypofractionated concurrent chemoradiotherapy, as well as the incidence rates of radiation oesophagitis and radiation pneumonitis (grade III and higher) and other grade III and above non-haematologic toxicities. However, fatigue, loss of appetite, nausea and vomiting were not included. The secondary endpoints are to observe the overall response rate, progression-free survival (PFS), median survival time (MST) and overall survival (OS). The survival time was measured from the initiation of the concurrent radiochemotherapy until death due to any cause or the last follow-up event. Only the first treatment failure was considered as the reason for failure. ${ }^{11}$

\section{Research design}

The present study is a prospective, multicenter, phase II, open-label exploratory study. The study will be conducted in three hospitals in China, including The Second Hospital of Hebei Medical University, North China Petroleum Bureau General Hospital, Hebei Medical University and No.1 Hospital of Shijiazhuang City, and enrol patients who are 18-75 years of age and who have been definitively diagnosed with NSCLC by pathological or cytological examinations. The patients who meet the inclusion criteria will receive concurrent chemoradiotherapy. This study has been approved by medical ethics committees: Research Ethics Committee of the Second Hospital of Hebei Medical University, Ethics Committee of the North China Petroleum Bureau General Hospital of Hebei Medical University and Medical Research Ethics Committee of the No.1 Hospital of Shijiazhuang City. 
Table 1 The maximum dose of radiation tolerated by normal tissues

\begin{tabular}{ll}
\hline Organ at risk & $\begin{array}{l}\text { Prespecified normal tissue } \\
\text { doses }\end{array}$ \\
\hline $\begin{array}{l}\text { Spinal canal PRV } \\
\begin{array}{l}\text { Lung (double gross } \\
\text { tumour volume) }\end{array}\end{array}$ & $\begin{array}{l}\text { Maximum dose } \leq 45 \mathrm{~Gy} \text {, and } \\
\text { each fraction } \leq 2 \mathrm{~Gy}\end{array}$ \\
& MLD $\leq 16 \mathrm{~Gy}$ \\
\hline Oesophagus & V5 $\leq 65 \%$ \\
Heart & Maximum dose $\leq 69 \mathrm{~Gy}$ \\
& Maximum dose $\leq 70 \mathrm{~Gy}$ \\
\hline Brachial plexus & Mean $\leq 30$ Gy \\
\hline
\end{tabular}

AJCC, American Joint Committee on Cancer; cT4, clinical staging tumor4; MLD, mean lung dose; PRV, planning organ at risk volume.

Patient and public involvement statement

Patients or the public were not involved in the design, conduct, reporting and dissemination plans of our research.

\section{Research implementation}

Patients will be comprehensively evaluated by thoracic surgeons, radiation oncologists, medical oncologists and related internists. Patients who meet the inclusion criteria will be fully informed of the details of the trial protocol by the radiation oncologists. The patients will be enrolled in the clinical trial after providing written informed consent. The details of this concurrent chemoradiotherapy regimen, the benefits and possible harms, the alternative regimens, ancillary and post-trial care, and the compensation to those who might suffer harm from trial participation etc are detailed listed in the Informed Consent. An English example of the model consent form is provided as an online supplemental file. The individualised radiotherapy dose will be determined based on the maximum irradiation tolerable dose of the normal tissues, including the lung, oesophagus, spinal cord, brachial plexus and heart (for details see tables 1 and 2). The maximum radiotherapy dose, namely, the prescription dose, is attained when the irradiation dose of any of the above normal tissues reaches the upper limit. If the irradiation dose of the normal tissues does not reach the upper limit, the maximum radiotherapy dose will be set to $69 \mathrm{~Gy}$ according to the results of our previous phase I trial. ${ }^{10}$ The trial duration for recruitment is expected for 3 years from 2019 to 2022. All the recruitment patients will be followed up for 5 years. For the first 2 years, they will be followed up every 3 months on the completion of concurrent chemoradiotherapy for the first 2 years. Then every half a year, to a maximum of up to 5 years. Follow-up includes physical examination, symptoms, blood routine, liver and kidney function, CT of the chest and CT, bone scan would be done if indicated clinically. Acute and late radiation toxicities continue to be recorded at each follow-up visit (according to the CTCAE V.5.0).

\section{Individualised radiotherapy prescription}

Radiotherapy doses all patients received are individually escalated until one or more of the OAR tolerance or the maximum dose of $69 \mathrm{~Gy}$ is reached. The patients will receive hypofractionated radiotherapy at $3 \mathrm{~Gy} /$ fraction. Individualised radiotherapy prescription is based on the irradiation doses of normal tissues (specified to a volume of $1 \mathrm{~cm}^{3}$ ). The total doses ranged from 45 to $69 \mathrm{~Gy}, 15$ to 23 fractions. The dose per fraction ( $3 \mathrm{~Gy} /$ fraction) is fixed and not allowed to be changed. The MTDof normal tissues are shown in table 1 . The maximum irradiation dose to the spinal cord is calculated in the concept of

\begin{tabular}{|c|c|c|c|c|}
\hline $\begin{array}{l}\text { Irradiated dose per fraction to } \\
\text { spinal canal PRV }\end{array}$ & Fractions & Maximum dose & $\begin{array}{l}\text { Integer number for } \\
\text { fractions }\end{array}$ & $\begin{array}{l}\text { Maximum dose of } \\
\text { EQD2 }\end{array}$ \\
\hline 2.1 & 21.01 & 44.12 & 21 & 44.1 \\
\hline 2.2 & 19.67 & 43.27 & 19 & 41.8 \\
\hline 2.3 & 18.46 & 42.45 & 18 & 41.4 \\
\hline 2.4 & 17.36 & 41.67 & 17 & 40.8 \\
\hline 2.5 & 16.36 & 40.91 & 16 & 40.0 \\
\hline 2.6 & 15.45 & 40.18 & 15 & 39.0 \\
\hline 2.7 & 14.62 & 39.47 & 14 & 37.8 \\
\hline 2.8 & 13.85 & 38.79 & 13 & 36.4 \\
\hline 2.9 & 13.15 & 38.14 & 13 & 37.7 \\
\hline 3.0 & 12.5 & 37.5 & 12 & 36 \\
\hline
\end{tabular}

1. This table is used when the dose per fraction irradiated to spinal canal PRV exceeds $2 \mathrm{~Gy}$. 2 . The $\alpha / \beta$ ratio is set to $3 \mathrm{~Gy},{ }^{34}{ }^{35}$ and the fractionated dose is subjected to rounding.

EQD2, equivalent dose in 2 Gy fractions; PRV, planning organ at risk volume. 
equivalent dose in $2 \mathrm{~Gy}$ fractions (EQD2) when the radiation exceeds $2 \mathrm{~Gy} /$ fraction (table 2 ).

\section{Inclusion criteria}

1. The patients will be newly diagnosed with NSCLC by pathological or cytological examinations. The NSCLC will be stage III (clinical stage is determined using the 8th ed. of the AJCC Cancer Staging Manual ${ }^{19}$ ), surgically unresectable (determined by a multidisciplinary team evaluation including thoracic surgeons, radiation oncologists, medical oncologists, pathologists and radiologists) and unsuitable for SBRT/stereotactic ablative radiotherapy (SABR). Patients with cT4 NSCLC who has invaded the oesophagus will be excluded.

2. The patient will be $\geq 18$ years and $\leq 75$ years of age.

3. The patients will have an estimated survival time of $\geq 3$ months.

4. The patients will have a Karnofsky Performance Scale (KPS) Score $\geq 70$.

5. The patients will have normal blood parameters and hepatorenal function.

6 . The patients will have no serious medical diseases that need hospitalisation.

7. The patients will have a $1 \mathrm{~s}$ volume of $\geq 0.75 \mathrm{~L}$ in the static lung function test. ${ }^{20}$

\section{Exclusion criteria}

1. Patients who are pregnant or lactating.

2. Patients who have received antitumor treatments.

3. Patients who have a history of malignant tumours.

4. Patients who have a KPS Score of $<70$ points.

5. Patients who have the following concurrent diseases: history of pulmonary fibrosis, myocardial infarction within 12 months, grade II and above heart failure, uncontrolled heart failure, uncontrolled chronic obstructive pulmonary disease and uncontrolled severe diabetes (evaluated by internal medicine specialists).

6. Patients who are unsuitable for hypofractionated concurrent chemoradiotherapy.

\section{Concurrent chemoradiotherapy regimens}

\section{Radiation therapy}

1. Fractionation mode: Moderate hypofractionation will be employed (3Gy/fraction). One fraction will be administered every day from Monday to Friday (five fractions per week). No radiotherapy will be given to the patients on weekends with the exception of radiotherapy interruption occurrence. The patient will be treated on the weekend as compensation when unexpected radiotherapy fractions missed. In the ideal condition, the first fraction will be delivered on Monday. If the ideal condition cannot be reached, the radiotherapy process will include as few weekends as possible.

2. Radiotherapy technique: Inverse intensity-modulated radiation therapy (IMRT) planning will be employed. Volumetric modulated arc therapy, RapidArc and fixed-field IMRT are all applicable. In terms of fixedfield IMRT, at least five fields will be used. Ideal dose distribution: $100 \%$ of the prescribed dose will cover at least $95 \%$ of the planning target volume (PTV), and the lowest and highest acceptable doses (specified to a volume of $1 \mathrm{~cm}^{3}$ ) will be $93 \%-107 \%$ of the prescribed dose. Cone-beam CT will be conducted a minimum of once per week.

3. Respiratory control: Four-dimensional CT and respiratory gating are not mandatory due to the lack of relevant equipment in the two centres participating in the study. The patients will be instructed before the radiotherapy to breathe as calmly as possible.

4. Radiotherapy implementation: The patients will assume a supine position with their hands clasped on top of their heads. A thermoplastic body cast or vacuum cushion will be employed to immobilise the patients and properly restrict respiratory motion. A spiral CT scan will be performed in the treatment position (plain scan+enhancement, $3 \mathrm{~mm}$ layer thickness). Scan range: The upper boundary will be set to the annular cartilage (in special cases, the entire neck will be included in the scan). The lower boundary will include intact lungs and the lower edge of the liver. Image data will be input into the IMRT planning system. The target areas will be outlined in the plain-scan image sequences, and the enhanced image sequences in the arteriovenous phase will be merged for reference.

5 . Delineation of the NSCLC target volumes ${ }^{21}$ : The target volumes in the primary pulmonary lesion will be delineated in the lung window $(-1600,600$ Hounsfield units (HU)), while the mediastinal target volumes will be delineated in the mediastinal window (400, $20 \mathrm{HU})$. Involved-field irradiation will be used, and elective nodal irradiation will be prohibited. ${ }^{16-18}$ The target volumes will be defined as follows. The gross tumour volume (GTV) will be defined as the primary pulmonary lesions and lymph nodes with a short diameter greater than $1 \mathrm{~cm}$ in the CT image or lymph nodes with a short diameter of $1 \mathrm{~cm}$ or less that are positive by positron emission tomography/CT or by biopsy pathology. The clinical target volume (CTV) will be defined as the GTV plus a margin of $6 \mathrm{~mm}$ (squamous cell carcinoma and non-adenocarcinomas) or $8 \mathrm{~mm}$ (adenocarcinoma). For metastatic lymph nodes, a margin of 3-5 mm will be added. The PTV will be defined as the CTV plus a margin of $10-15 \mathrm{~mm}$, partly referring on the degree of respiratory movement observed in the simulator if it is applicable. The GTV will be determined by two radiation oncologists and one radiologist.

6. Delineation of normal tissues and organs: Radiotherapy physicists will outline body surface contours and OAR according to the RTOG standard. ${ }^{22}$ Radiation oncologists will then review the delineation, ensuring that no layer of oesophagus is included in the PTV. ${ }^{11}$

\section{Radiotherapy quality control}

Radiotherapy-related data of all patients in each centre should be uploaded to a database created by the PI's organisation and managed by specific personnel. The 
data will include general clinical conditions, image data of the delineated target volumes, radiotherapy prescription, radiotherapy dose distribution, plan verification, adverse effects (AEs) and the management of AE, etc. The first 1-3 patients in each subcentre will be reviewed by the study chair and the chief medical physicists at the PI's organisation. If no major deviations are detected, random inspection will be conducted on the treatment regimens for the remaining patients. If any major deviation is found in the treatment protocols of the first 1-3 patients, a preaudit system will be implemented. The radiotherapy plans developed by each centre will first be reviewed and/or modified by the PI and the chief medical physicists at the PI's organisation. Radiotherapy will only be administered after the protocol requirements are met. If three consecutive cases are found to be compliant with the requirements, a preaudit will not be performed on the rest of the patients. The treatment data uploaded by each centre will be subjected to a retrospective audit. Study chair is responsible for access to the final trial dataset, and disclosure of contractual agreements.

\section{Chemotherapy}

Platinum-containing

docetaxel+lobaplatin.

$$
\text { dual-drug regimen: }
$$

The regimen will be administered as follows: docetaxel, $60 \mathrm{mg} / \mathrm{m}^{2}, \mathrm{~d} 1$; lobaplatin, $30 \mathrm{mg} / \mathrm{m}^{2}$, $\mathrm{d} 1$; repeated every 28 days. The first cycle of chemotherapy will begin on the first day of radiotherapy.

Up to four cycles of consolidation chemotherapy will be performed after completion of the radiotherapy, using the same regimen as above.

Docetaxel doses are derived from Asian studies, ${ }^{23} 24$ while lobaplatin doses are derived from phase I-III clinical trials conducted in Chinese patients with NSCLC. ${ }^{25} 26$

\section{Other therapy}

Induced chemotherapies are permitted before the start of chemoradiotherapy and any other antitumour therapies are not permitted before the start and during of chemoradiotherapy. Supportive care to ensure the implementation of the chemoradiotherapy regimen is permitted during the treatment, including nutritional support via intravenous rehydration, use of GSF and antioesophagitis therapies, etc. Once progression disease happens during or after the completion of this concurrent chemoradiotherapy regimen of this trial or patients are withdrawn from this trial because of any reasons, subsequent antitumour therapies are permitted. The treatments are individualised decided by physicians in the trial centres.

\section{Dose attenuation}

Dose attenuations are implemented based on the most serious adverse events that occurred at any point after the start of treatment. Nonhaematological toxicities of grade III or higher occur (with the exception of grade III nausea, vomiting or weight loss), radiotherapy will be postponed until the toxicity will be resolved. In contrast, if adverse events occur that are unrelated to radiotherapy, such as peripheral neuropathy, the radiotherapy is continued but chemotherapy will be suspended. The chemotherapy is resumed after these adverse events dissipate. The following chemotherapy dose attenuation procedures are employed. In the event of grade III or grade IV thrombocytopenia, grade III or grade IV anaemia, grade IV neutropaenia, or grade III or grade IV nonhaematologic toxicities (except for grade III nausea, vomiting or weight loss), both radiotherapy and chemotherapy will be suspended until the toxicity are resolved. If the toxicity cannot be resolved within 2 weeks, the patient is withdrawn from the study. The docetaxel and lobaplatin doses of this patient's subsequent chemotherapy cycle will be reduced by $25 \%$, and the patient will receive prophylactic GSF treatment. If a patient exhibit grade III neutropaenia or grade II thrombocytopenia, chemotherapy is stopped but radiotherapy is continued. The docetaxel and lobaplatin doses of this patient's subsequent chemotherapy cycle remain unchanged, and the patient will receive prophylactic G-CSF (granulocyte colony-stimulating factor) treatment. ${ }^{10}$

\section{Statistical design}

The Simon design is employed in our phase II study. ${ }^{27}$ The primary endpoint is safety, including determining the incidence rates of severe radiation-induced oesophagitis and pneumonitis. Therefore, oesophageal and pulmonary toxicity will be simultaneously considered. An acceptable incidence rate of grade III and higher radiation oesophagitis will be $15 \%$, whereas an incidence rate of $30 \%$ will not be acceptable. The acceptable incidence rate of grade III and higher radiation pneumonitis will be $8.5 \%$, whereas an incidence rate of $22.5 \%$ will be unacceptable. The statistical power will be $85 \%$, and the $\alpha$ value will be set at 0.15 . Based on the above standards, the sample number, calculated using PASS V.11.0, will be 30 .

The present study is a two-stage phase II clinical trial. ${ }^{27}$ The following situations will result in early termination of the trial:

1. In the first stage, 12 patients will be enrolled. The following situations will result in early termination of the trial:

1. Occurrence of radiation pneumonitis of grade III and higher in $4 / 12$ or more patients.

2. Occurrence of radiation oesophagitis of grade IV and higher in 4/12 or more patients, and/or grade III radiation oesophagitis causes an interruption of the radiotherapy for more than 1 week.

3. Patients in each centre who develop the conditions described in 'A' or 'B' will be reported to the PI of the group leader's organisation for confirmation. Grade III radiation oesophagitis may not show dose-limiting toxicity. When grade III radiation oesophagitis occurs, clinical intervention will be actively carried out to minimise its impaction radiotherapy and avoid an interruption in radiotherapy for more than 7 days. 
2. The second stage: If early termination does not occur at 3 months after the last patient enrolled in the first stage completes radiotherapy, 18 more patients will be enrolled to further examine toxicity and efficacy. A total of 30 patients will be enrolled.

The second endpoints, including PFS, MST and OS, will be analysed using the SPSS V.19.0 bio-statistical software package. The survival data will be evaluated using the Kaplan-Meier method.

Study chair (X-YX) will decide the interim analyses (the first stage of this trial) and make the final decision to terminate the trial if applicable.

\section{ETHICS AND DISSEMINATION}

The study has been approved by medical ethics committees: Research Ethics Committee of the Second Hospital of Hebei Medical University, Ethics Committee of the North China Petroleum Bureau General Hospital affiliated to Hebei Medical University and Medical Research Ethics Committee of the No.1 Hospital of Shijiazhuang. The trial is conducted in accordance with the Declaration of Helsinki. The trial results will be actively disseminated through academic conference presentations and peerreviewed publications.

Individual participant data (IPD) sharing statement:

Plan to share IPD: After the publication of this study, we could share the IPD. However, this sharing is limited to academic research.

Person to be contacted: Study chair, X-YX.

Contact information: zyy_lq@petrochina.com.cn

\section{DISCUSSION}

It is generally believed that NSCLC cells begin to undergo accelerated repopulation in the 3rd-4th weeks of radiotherapy. Therefore, radiotherapy of more than 4 weeks has a reduced killing effect on tumour cells. ${ }^{8}$ Shortening the total treatment time of radiotherapy is one of the key factors to improving the efficacy.

Accelerated radiotherapy, that is, capable of shortening the total treatment duration generally has two modes. The first, hyperfractionated radiotherapy, is represented by continuous hyperfractionated accelerated radiotherapy (CHART) ${ }^{28}$ and CHART weekend less $^{29}$ and has achieved good clinical outcomes. The second, hypofractionated radiotherapy, is represented by the SOCCAR trial ${ }^{16}$ and a study conducted in the Netherlands. ${ }^{17}$ Both studies used a single-fractionation dose of $2.75 \mathrm{~Gy}$ and achieved good survival results. However, most of the published studies to date and ongoing clinical trials adopted a uniform prescription dose. Namely, all patients were given the same radiotherapy dose, regardless of the tumour size and adjacent location to vital organs. We have entered the era of precision treatment. Stage III NSCLC is a heterogeneous disease characterised by substage differences (IIIA, IIIB and IIIC), lymph node N2 classification (single or multiple fusions), tumour location and adjacency to vital organs. To treat tumours with high degrees of heterogeneity, individualised radiotherapy should be administered. However, the criteria for determining the individualised prescription doses remain to be defined in clinical practice. The MAASTRO group proposed the concept of 'isotoxicity' in hyperfractionated therapy. Namely, patients are given different radiotherapy doses according to the maximum radiotherapy dose that can be tolerated by their vital organs. The maximum radiotherapy doses tolerated to the normal tissues are preseted. The highest radiotherapy dose within this range is administered to each patient. The series of studies conducted by the MAASTRO group, which included a model study, feasibility study and long-term follow-up of survival, all proved that 'isotoxic' prescription doses were safe and effective. ${ }^{30-32}$ However, hypofractionation has radiobiological effects different from hyperfractionation, and the currently used so-called normal tissue limits are mostly derived from conventional fractionated irradiation. There is currently no evidence whether the 'isotoxicity' concept is suitable for hypofractionated irradiation.

The National Comprehensive Cancer Network guidelines clearly state that the physical dose of radiation tolerable to normal tissues declines as the singlefraction dose in SBRT/SABR increases. ${ }^{33}$ Because the single-fraction dose is very high in SBRT/SABR (up to $22 \mathrm{~Gy} /$ fraction) and the modified SBRT/SABR uses a single-fraction dose of 7-10 Gy, normal tissue limits are strictly enforced in SBRT/SABR. However, the radiobiological effect of SBRT/SABR is different from that of moderate hypofractionation. Therefore, direct application of the SBRT/SABR-derived normal tissue limits in moderately hypofractionated radiotherapy may cause difficulty in dose escalation.

There are no definitive data regarding moderately hypofractionated radiotherapy, especially when it is combined with concurrent chemotherapy. Therefore, using the 'isotoxicity' concept of hyperfractionation and certain hypofractionated radiotherapy studies as reference, we propose a regimen based on the MTD in normal tissues (tables 1 and 2). We plan to conduct this phase II clinical trial to verify the safety of our regimen. For certain organs, such as the spinal cord, we convert the radiation dose received to EQD2 to ensure its safety to the maximum extent possible. ${ }^{345}$

If our hypothesis is confirmed in the phase II trial, isotoxic, individualised radiotherapy regimens will be examined in phase III randomised controlled trials and compared with conventional fractionated irradiation, which will provide high-level evidence for concurrent moderately hypofractionated radiotherapy and chemotherapy.

Contributors Y-EL participated in the design of the subject, drafted the manuscript and will analyse the clinical data. $X-Y X$ guided the design of the subject, and helped to draft the manuscript. RZ participated in the design of the subject and will 
analyse the clinical data. X-JC participated in the design of the subject, and will carry out the clinical implementation of the trial. Y-XD participated in the design of the subject, and will carry out the clinical implementation of the trial. C-XL participated in the design of the subject. Y-LQ participated in the design of the subject and will carry out the clinical implementation of the trial. W-QL participated in the design of the subject and will carry out the clinical implementation of the trial. X-CR participated in the design of the subject and will carry out the clinical implementation of the trial. QL (the corresponding author) was the PI of this 'study protocol', who designed the subject and helped to draft the manuscript.

Funding Our study is funded by Health Commission of Hebei Province (China, http://www.hebwsjs.gov.cn/), which belongs to the government of Hebei Province, China. The funding number is G2018068. We declare that we have never received any funding from tobacco companies.

Competing interests None declared.

Patient consent for publication Not required.

Provenance and peer review Not commissioned; externally peer reviewed.

Supplemental material This content has been supplied by the author(s). It has not been vetted by BMJ Publishing Group Limited (BMJ) and may not have been peer-reviewed. Any opinions or recommendations discussed are solely those of the author(s) and are not endorsed by BMJ. BMJ disclaims all liability and responsibility arising from any reliance placed on the content. Where the content includes any translated material, BMJ does not warrant the accuracy and reliability of the translations (including but not limited to local regulations, clinical guidelines, terminology, drug names and drug dosages), and is not responsible for any error and/or omissions arising from translation and adaptation or otherwise.

Open access This is an open access article distributed in accordance with the Creative Commons Attribution Non Commercial (CC BY-NC 4.0) license, which permits others to distribute, remix, adapt, build upon this work non-commercially, and license their derivative works on different terms, provided the original work is properly cited, appropriate credit is given, any changes made indicated, and the use is non-commercial. See: http://creativecommons.org/licenses/by-nc/4.0/.

\section{ORCID iD}

Qiang Lin http://orcid.org/0000-0001-9599-4121

\section{REFERENCES}

1 Bray F, Ferlay J, Soerjomataram I, et al. Global cancer statistics 2018: GLOBOCAN estimates of incidence and mortality worldwide for 36 cancers in 185 countries. CA Cancer J Clin 2018;68:394-424.

2 Aupérin A, Le Péchoux C, Rolland E, et al. Meta-Analysis of concomitant versus sequential radiochemotherapy in locally advanced non-small-cell lung cancer. J Clin Oncol 2010;28:2181-90.

3 Bradley JD, Paulus R, Komaki R, et al. Standard-Dose versus highdose conformal radiotherapy with concurrent and consolidation carboplatin plus paclitaxel with or without cetuximab for patients with stage IIIA or IIIB non-small-cell lung cancer (RTOG 0617): a randomised, two-by-two factorial phase 3 study. Lancet Oncol 2015;16:187-99.

4 Martel MK, Ten Haken RK, Hazuka MB, et al. Estimation of tumor control probability model parameters from 3-D dose distributions of non-small cell lung cancer patients. Lung Cancer 1999;24:31-7.

5 Onishi H, Shirato H, Nagata $Y$, et al. Hypofractionated stereotactic radiotherapy (HypoFXSRT) for stage I non-small cell lung cancer: updated results of 257 patients in a Japanese multi-institutional study. J Thorac Oncol 2007;2:S94-100.

6 Bradley JD, Moughan J, Graham MV, et al. A phase I/II radiation dose escalation study with concurrent chemotherapy for patients with inoperable stages I to III non-small-cell lung cancer: phase I results of RTOG 0117. Int J Radiat Oncol Biol Phys 2010;77:367-72.

7 Bayman N, Blackhall F, McCloskey P, et al. How can we optimise concurrent chemoradiotherapy for inoperable stage III non-small cell lung cancer? Lung Cancer 2014;83:117-25.

8 Partridge M, Ramos M, Sardaro A, et al. Dose escalation for nonsmall cell lung cancer: analysis and modelling of published literature. Radiother Oncol 2011;99:6-11.

9 Ohri N, Dicker AP, Lawrence YR. Can drugs enhance hypofractionated radiotherapy? a novel method of modeling radiosensitization using in vitro data. Int $J$ Radiat Oncol Biol Phys 2012;83:385-93.

10 Lin Q, Liu Y-E, Ren X-C, et al. Dose escalation of accelerated hypofractionated three-dimensional conformal radiotherapy (at $3 \mathrm{~Gy} /$ fraction) with concurrent vinorelbine and carboplatin chemotherapy in unresectable stage III non-small-cell lung cancer: a phase I trial. Radiat Oncol 2013;8:201.

11 Ren X-C, Wang Q-Y, Zhang R, et al. Accelerated hypofractionated three-dimensional conformal radiation therapy (3 Gy/fraction) combined with concurrent chemotherapy for patients with unresectable stage III non-small cell lung cancer: preliminary results of an early terminated phase II trial. BMC Cancer 2016;16:288.

12 Haslett K, Franks K, Hanna GG, et al. Protocol for the isotoxic intensity modulated radiotherapy (IMRT) in stage III non-small cell lung cancer (NSCLC): a feasibility study. BMJ Open 2016;6:e010457.

13 Landau DB, Hughes L, Baker A, et al. IDEAL-CRT: a phase 1/2 trial of isotoxic dose-escalated radiation therapy and concurrent chemotherapy in patients with stage II/III non-small cell lung cancer. Int J Radiat Oncol Biol Phys 2016;95:1367-77.

14 Fleming C, Cagney DN, O'Keeffe S, et al. Normal tissue considerations and dose-volume constraints in the moderately hypofractionated treatment of non-small cell lung cancer. Radiother Oncol 2016;119:423-31.

15 Thirion P, Holmberg O, Collins CD, et al. Escalated dose for non-small-cell lung cancer with accelerated hypofractionated three-dimensional conformal radiation therapy. Radiother Oncol 2004;71:163-6.

16 Maguire J, Khan I, McMenemin R, et al. SOCCAR: a randomised phase II trial comparing sequential versus concurrent chemotherapy and radical hypofractionated radiotherapy in patients with inoperable stage III non-small cell lung cancer and good performance status. Eur J Cancer 2014;50:2939-49.

17 Walraven I, van den Heuvel M, van Diessen J, et al. Long-Term follow-up of patients with locally advanced non-small cell lung cancer receiving concurrent hypofractionated chemoradiotherapy with or without cetuximab. Radiother Oncol 2016;118:442-6.

18 Westover KD, Loo BW, Gerber DE, et al. Precision Hypofractionated radiation therapy in poor performing patients with non-small cell lung cancer: phase 1 dose escalation trial. Int J Radiat Oncol Biol Phys 2015;93:72-81.

19 Rami-Porta R, Travis WD, Asamura H, et al. Ajcc cancer staging manual. 8th edn. Springer International Publishing, 2017: 431-56.

20 Cho KH, Ahn SJ, Pyo HR, et al. A phase II study of synchronous three-dimensional conformal boost to the gross tumor volume for patients with unresectable stage III non-small-cell lung cancer: results of Korean radiation Oncology Group 0301 study. Int J Radiat Oncol Biol Phys 2009;74:1397-404.

21 Zhu GY, Xia TY, Wang LH, et al. Consensus and controversies on delineation of radiotherapy target volume for patients with non-small cell lung cancer. Chines Journal of Radiation Oncology 2008;17:432-6. (in Chinese).

22 Available: https://www.rtog.org/ CoreLab/ Contouring Atlases/ LungAtlas.aspx [Accessed 12 Dec 2018]

23 Lee JJ, Han J-Y, Lee DH, et al. A phase II trial of docetaxel plus capecitabine in patients with previously treated non-small cell lung cancer. Jpn J Clin Oncol 2006;36:761-7.

24 Lai C-L, Tsai C-M, Chiu C-H, et al. Phase II randomized trial of tri-weekly versus days 1 and 8 Weekly docetaxel as a second-line treatment of advanced non-small cell lung cancer. Jpn J Clin Oncol 2005;35:700-6.

25 Qin SK, Cheng Y, Chen ZD, et al. Dose escalation trial of lobapatin combined with paclitaxel in patients with advanced non-small cell lung cancers as the first-line therapy. Chinese Clinical Oncology 2018;23:97-100. (in Chinese).

26 Qin SK, Cheng Y, Li J, et al. A randomized, controlled, muticenter phase III study to compared efficacy and safety of lobaplatin plus paclitaxel versus carboplatin plus paclitaxel as the first-line therapy in Chinese patients with advanced non-small cell lung cancer. Chinese Clinical Oncology 2018;23:193-9. (in Chinese).

27 Simon R. Optimal two-stage designs for phase II clinical trials. Control Clin Trials 1989;10:1-10.

28 Saunders M, Dische S, Barrett A, et al. Continuous, hyperfractionated, accelerated radiotherapy (CHART) versus conventional radiotherapy in non-small cell lung cancer: mature data from the randomised multicentre trial. Radiotherapy and Oncology 1999;52:137-48.

29 Saunders MI, Rojas A, Lyn BE, et al. Experience with dose escalation using CHARTWEL (continuous hyperfractionated accelerated radiotherapy weekend less) in non-small-cell lung cancer. $\mathrm{Br} J$ Cancer 1998;78:1323-8.

30 van Baardwijk A, Bosmans G, Bentzen SM, et al. Radiation dose prescription for non-small-cell lung cancer according to normal tissue dose constraints: an in silico clinical trial. Int J Radiat Oncol Biol Phys 2008;71:1103-10.

31 van Baardwijk A, Bosmans G, Boersma L, et al. Individualized radical radiotherapy of non-small-cell lung cancer based on normal tissue 
dose constraints: a feasibility study. Int $J$ Radiat Oncol Biol Phys 2008;71:1394-401.

32 van Baardwijk A, Wanders S, Boersma L, et al. Mature results of an individualized radiation dose prescription study based on normal tissue constraints in stages I to III non-small-cell lung cancer. J Clin Oncol 2010;28:1380-6.

33 National comprehensive cancer network. Available: https://www. nccn.org/professionals/physician_gls/pdf/nscl.pdf [Accessed 3 Mar 2019].
34 Cannon DM, Mehta MP, Adkison JB, et al. Dose-Limiting toxicity after hypofractionated dose-escalated radiotherapy in non-small-cell lung cancer. J Clin Oncol 2013;31:4343-8.

35 Osti MF, Agolli L, Valeriani M, et al. Image guided Hypofractionated 3-dimensional radiation therapy in patients with inoperable advanced stage non-small cell lung cancer. Int $J$ Radiat Oncol Biol Phys 2013;85:e157-63. 